\title{
Schwerer Verlauf einer Leptospirose mit akutem Nierenversagen und ausgeprägtem Ikterus (Morbus Weil)
}

\author{
W. F. Notheis, B. K. Krämer, H.-G. Leser, J. Rüschoff, E. P. Kromer und A. J. G. Riegger \\ Klinik und Poliklinik für Innere Medizin II (Direktor: Prof. Dr. A. J. G. Riegger), Klinik und Poliklinik für Innere Medizin I \\ (Direktor: Prof. Dr. J. Schölmerich) und Pathologisches Institut (Direktor: Prof. Dr. F. Hofstädter) der Universität \\ Regensburg
}

\begin{abstract}
Bei einem 77jährigen Patienten kam es zu unklarem Fieber bis $38,4^{\circ} \mathrm{C}$, Diarrhoen, akutem Nierenversagen (Kreatinin bis $8,7 \mathrm{mg} / \mathrm{dl}$; Harnstoff bis $308 \mathrm{mg} / \mathrm{dl}$ ) und schwerem Ikterus (Gesamtbilirubin bis $24,3 \mathrm{mg} / \mathrm{dl}$ ). Zudem wies er eine Thrombozytopenie, Konjunktivitis und Epistaxis auf, und es entwickelten sich zerebrale Symptome mit Somnolenz und allgemeiner Verlangsamung. Da zunächst eine Cholangitis bei bekannten Gallensteinen vermutet worden war, hatte der Patient eine antibiotische Therapie mit Ampicillin zwei$\mathrm{mal} 2 \mathrm{~g} / \mathrm{d}$, und Metronidazol, zweimal $0,5 \mathrm{~g} / \mathrm{d}$, erhalten; daraufhin ging das Fieber zurück. Wegen des Nierenversagens waren Hämodialyse bzw. Hämofiltration unter strenger Flüssigkeitskontrolle erforderlich. Endoskopisch konnte ein Verschlußikterus ausgeschlossen werden; es wurde jedoch der Verdacht auf eine entzündliche Lebererkrankung bzw. differentialdiagnostisch eine Leberzirrhose geäußert. Die serologische Untersuchung ergab erhöhte Titer für Leptospira interrogans var. sejroe $(1: 200$, später $1: 1600)$. Der histologische Befund einer Leberbiopsie war vereinbar mit einer Leptospirose. Aufgrund der hohen Entzündungsaktivität in der Leber erhielt der Patient dreimal täglich 2 Mega-E Penicillin G für 6 Tage. Im weiteren Verlauf besserte sich die Ausscheidungsfunktion, und der Ikterus war rückläufig. Bei der Entlassung am 36. Tag war der Patient wieder in gutem Allgemeinzustand bei noch leicht erhöhten Kreatinin- und Bilirubinwerten (jeweils $1,7 \mathrm{mg} / \mathrm{dl}$ ).
\end{abstract}

Die Leptospirose gilt weltweit als die am weitesten verbreitete Zoonose; sie ist auf allen Kontinenten beheimatet, kommt jedoch häufiger in tropischen als in gemäßigten Regionen vor. Nach den letzten Daten des Statistischen Bundesamtes lag die

Dtsch. med. Wschr. 118 (1993), 1437-1441

(c) Georg Thieme Verlag Stuttgart - New York

\section{A severe case of leptospirosis with acute renal failure and marked jaundice (Weil's disease)}

A 77-year-old man developed a fever up to $38.4^{\circ} \mathrm{C}$, with diarrhoea, acute renal failure (creatinine up to $8.7 \mathrm{mg} / \mathrm{dl}$; urea up to $308 \mathrm{mg} / \mathrm{dl}$ ) and marked jaundice (total bilirubin up to 24.3 $\mathrm{mg} / \mathrm{dl}$ ). In addition there was thrombocytopenia, conjunctivitis and epistaxis, as well as cerebral symptoms with somnolence and general slowing up. At first he was thought to have cholangitis resulting from previously diagnosed gall-stones, and he was therefore treated with ampicillin, $2 \mathrm{~g}$ two times daily, and metronidazole, $0.5 \mathrm{~g}$ two times daily. The fever regressed, but the renal failure required haemodialysis and haemofiltration under strict fluid control. Endoscopy excluded obstructive jaundice, but a suspicion of inflammatory liver disease or possibly cirrhosis was raised in the differential diagnosis. Serology revealed an increased titre for Leptospira interrogans var. sejroe (1: 200, later 1 : 1600). Liver biopsy finding was compatible with the diagnosis of leptospirosis. Because of the high inflammatory activity in the liver, 2 mega units of penicillin $G$ were administered three times daily for six days. Gradually the renal functions and jaundice improved and, on discharge on the 36th day, the patient was again in generally good health, although creatinine and bilirubin values were still slightly elevated $(1.7$ $\mathrm{mg} / \mathrm{dl}$ each).

Inzidenz der Leptospirosen für die Jahre 1986 bis 1990 in den alten Bundesländern zwischen 12 Krankheitsfällen im Jahre 1986 und 25 Krankheitsfällen im Jahre 1990; die Mehrzahl der Erkrankungen der letzten 3 Jahre wurden als Morbus Weil, die schwere Form der Leptospirose, gemeldet (14). 
Die Erregerspezies wird in zwei Gruppen unterteilt: Bei der Biflexa-Gruppe handelt es sich um harmlose Saprophyten, bei der InterrogansGruppe dagegen um humanpathogene Keime $(1,7$, $8,12,13)$.

Wir berichten über einen schweren Verlauf einer Leptospireninfektion, hervorgerufen durch die Serogruppe Leptospira interrogans varietas sejroe.

\section{Kasuistik}

\section{Anamnese}

Die stationäre Aufnahme des 77jährigen Patienten erfolgte zunächst in einer auswärtigen Klinik wegen seit 2 Tagen zunehmender Verschlechterung des Allgemeinzustandes bei Temperaturen bis $38,4^{\circ} \mathrm{C}$ und Diarrhoen mit deutlichen Zeichen der Exsikkose im hypovolämischen Schock (systolischer Blutdruck $50 \mathrm{~mm} \mathrm{Hg}$ ). An Vorerkrankungen waren eine absolute Arrhythmie bei chronischem Vorhofflimmern, ein kompletter Rechtsschenkelblock sowie eine arterielle Hypertonie bekannt. Darüber hinaus bestand der Verdacht auf eine koronare Herzkrankheit.

Trotz Ausgleichs des Flüssigkeitshaus haltes entwickelte sich ein akutes Nierenversagen mit Kreatininwerten um $8,7 \mathrm{mg} / \mathrm{dl}$ und Harnstoffwerten bis $308 \mathrm{mg} / \mathrm{dl}$, das eine tägliche Hämodialyse ab dem 1. Tag erforderlich machte. Des weiteren mußte wegen rezidivierenden Nasenblutens aufgrund von Erosionen am Locus Kiesselbachii mehrmals tamponiert werden. Ab dem 3. Aufenthaltstag zeigte sich ein zunehmender Ikterus mit acholischen Stühlen und dunkelbraunem Urin bei massiv erhöhtem direkten Bilirubin bis $19,8 \mathrm{mg} / \mathrm{dl}$ (Gesamtbilirubin $24,3 \mathrm{mg} / \mathrm{dl}$ ). Unter dem Verdacht auf eine Cholangitis bei Cholezystolithiasis erhielt der Patient zweimal täglich $2 \mathrm{~g}$ Ampicillin und $500 \mathrm{mg}$ Metronidazol. Mehrmalige Versuche einer Sondierung der Papille Vateri zum Ausschluß eines Verschlußikterus verliefen erfolglos. Am 10. Tag wurde der Patient daher zur weiteren Diagnostik und Therapie in unsere Klinik verlegt.

\section{Untersuchungsbefund}

Bei der Aufnahme auf unserer Intensivstation befand sich der etwa $100 \mathrm{~kg}$ schwere Patient (Größe $180 \mathrm{~cm}$ ) in schlechtem Allgemeinzustand; er war zeitlich und örtlich orientiert, jedoch deutlich somnolent und verlangsamt. Er wies einen ausgeprägten Haut- und Sklerenikterus auf. Typische Hautveränderungen im Sinne eines chronischen Leberschadens (Spider naevi, Palmarerythem oder Bauchglatze) bestanden nicht. Die Haut war trocken und wies mehrere Kratzspuren auf. Die Auskultation und Perkussion der Lunge und des Herzens ergaben unauffällige Befunde. Der Blutdruck lag bei 130/60 mm Hg, der Puls war arrhythmisch, ohne Defizit, mit Frequenzen um $80 / \mathrm{min}$. Bei der Untersuchung des Abdomens fanden sich weiche Bauchdecken ohne pathologische Resistenzen. Die Leber war perkutorisch in der rechten Medioklavikularlinie etwa $14 \mathrm{~cm}$ groß und am Rippenbogen tastbar. Die Milz konnte nicht getastet werden. Der Lymphknotenstatus war unauffällig. An beiden Beinen bestand eine ausgeprägte Varikosis bis hin zur Leistenregion mit diskreten prätibialen Ödemen. Der neurologische Status war unauffällig. Die Temperatur betrug $37,7^{\circ} \mathrm{C}$. Es bestand eine Konjunktivitis rechts.

\section{Klinisch-chemische Befunde}

Folgende Parameter waren pathologisch verändert: Leukozyten $(10900 / \mu l)$, Erythrozyten $(2,9 \mathrm{Mio} / \mu \mathrm{l})$, Hämoglobin $(9,0 \mathrm{~g} / \mathrm{dl})$, Hämatokrit $(27,7 \%)$ und Thrombozyten $(67000 / \mu l)$, Kreatinin $(8,7 \mathrm{mg} / \mathrm{dl})$, Harnstoff $(308 \mathrm{mg} / \mathrm{dl})$, Lactatdehydrogenase (272 U/l), Gesamtbilirubin (24,3 mg/dl, direktes Bilirubin 19,8 mg/dl), C-reaktives Protein $(30,9 \mathrm{mg} / \mathrm{dl})$, Blutsenkungsgeschwindigkeit $(50 / 65 \mathrm{~mm})$ und Lipase (369 U/l). Die Elektrolytbestimmungen waren mit $137 \mathrm{mmol} / \mathrm{l}$ für Natrium und $4,67 \mathrm{mmol} / \mathrm{l}$ für Kalium im Normbereich, die Calciumkonzentration war mit $1,79 \mathrm{mmol} / \mathrm{l}$ erniedrigt, die Phosphatkonzentration mit $3,74 \mathrm{mmol} / \mathrm{l}$ deutlich erhöht. Unauffällig waren die Werte für GOT (13 U/1), GPT (14 U/1) und alkalische Phosphatase (119U/1). Die Gerinnungsparameter zeigten bis auf eine mäßige Verlängerung der partiellen Thromboplastinzeit auf 46,3 s keinerlei Normabweichungen (Quick-Wert $83 \%$, Thrombinzeit $19,8 \mathrm{~s}$ ). Im Differentialblutbild zeigte sich eine relative Vermehrung der Granulozyten (87\%) bei Verminderung der Lymphozyten $(8 \%)$ und Normalverteilung der übrigen Zellen. Im Urinstatus wurden eine Leukozyturie $(500 / \mu \mathrm{l})$, Erythrozyturie $(250 / \mu \mathrm{l})$, Proteinurie $(75 \mathrm{mg} / \mathrm{dl})$ sowie eine Glucosurie $(50$ $\mathrm{mg} / \mathrm{dl}$ ) und Bilirubinurie $(3 \mathrm{mg} / \mathrm{dl}$ ) festgestellt.

Serologische Untersuchungen ergaben keine Hinweise auf eine frische Infektion mit Hepatitisvirus A, B oder Cytomegalieviren, Adenoviren, humanes Herpesvirus 6, Epstein-Barr- und Herpessimplex-Viren sowie mit Toxoplasmen, Yersinien, Borrelien und Brucellen. Deutlich erhöhte Antikörpertiter wurden für Leptospira canicola $(1: 400$, im weiteren Verlauf unverändert) und für Leptospira sejroe $(1: 200$, im weiteren Verlauf $1: 1600)$ gemessen.

In mehreren Blutkulturen, im Urin und im Stuhl ließen sich keine pathogenen Keime nachweisen. 


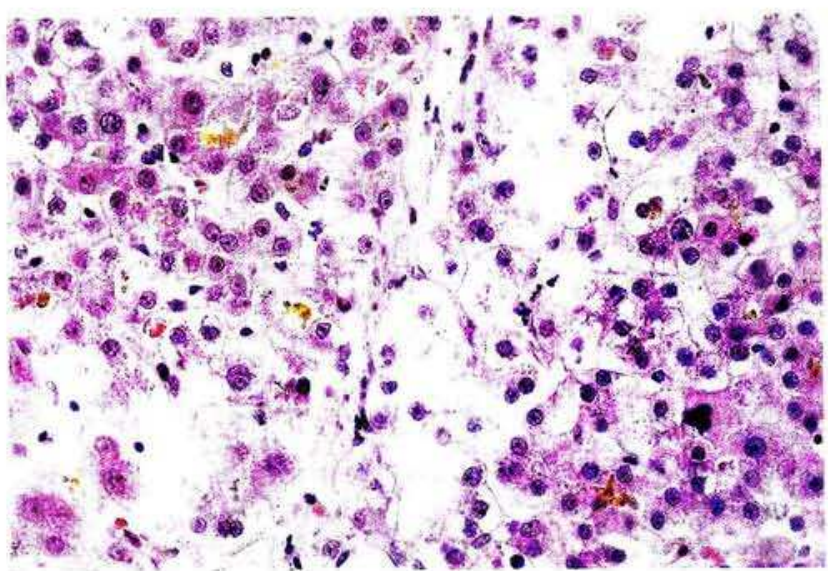

Abb. 1 Histologischer Schnitt des Leberbiopsates: deutliche hepatozelluläre und kanalikuläre Cholestase, geringgradige Vermehrung der KupfferSternzellen mit nur einzelnen intrasinusoidalen Granulozyten. HämatoxylinEosin, 200:1

\section{Apparative Verfahren}

Die Abdomensonographie ergab eine Hepatosplenomegalie mit homogener Verdichtung der Leber, im Sinne einer chronischen Hepatopathie. In der Gallenblase sah man mehrere kleine Konkremente im Bereich des Infundibulums. Der Ductus choledochus war mit 8 bis $9 \mathrm{~mm}$ grenzwertig weit ohne intraduktalen Steinnachweis. Die intrahepatischen Gallenwege waren nicht gestaut. Die Nieren zeigten eine altersentsprechende Größe und Parenchymstruktur.

Die Computertomographie des Abdomens bestätigte den sonographischen Befund. Die Leber wies abgestumpfte Ränder und eine diskret wellige Oberfläche auf, vereinbar sowohl mit einer Zirrhose als auch mit einem entzündlichen Prozeß. Die Milz war etwas vergrößert, und die Nieren waren unauffällig.

Das Röntgenbild des Thorax im Liegen ließ keine pneumonischen Infiltrate oder kardialen Stauungszeichen erkennen. Sichtbar war ein Zwerchfellhochstand rechts mit Abstumpfung des lateralen Rippenzwerchfellwinkels; dieser Befund entsprach - in Kenntnis eines subdiaphragmal gelegenen Granatsplitters - am wahrscheinlichsten einer Verschwielung.

Im Elektrokardiogramm waren ein überdrehter Rechtstyp, eine absolute Arrhythmie mit Frequenzen um 90/min sowie ein kompletter Rechtsschenkelblock und gehäufte ventrikuläre Extrasystolen zu erkennen.

Bei der endoskopischen retrograden Cholangiopankreatographie (ERCP) stellte sich ein diskret erweiterter Ductus choledochus ohne Hinweis auf Choledocholithiasis dar. Nachgewiesen wurden ferner die bekannten Gallenblasensteine mit einer Größe bis zu $8 \mathrm{~mm}$ sowie korkenzieherartige Verläufe intrahepatischer Gallengangsäste als möglicher Hinweis auf Regeneratknoten bei Verdacht auf Leberzirrhose.

\section{Therapie und Verlauf}

Aufgrund der Ergebnisse der serologischen Untersuchungen wurde eine akute Leptospirose (Morbus Weil) diagnostiziert. Da durch Endoskopie, Computertomographie und Sonographie des Abdomens der Verdacht auf eine Leberzirrhose erhoben wurde, führten wir 8 Tage nach der Aufnahme (Thrombozytenzahl zu diesem Zeitpunkt $155000 / \mu l)$ eine Leberpunktion durch. Die histologische Begutachtung ergab jedoch keine Leberzirrhose. Vielmehr bestand eine mittelgradige chronische, aktive Hepatitis mit ausgeprägter hepatozellulärer und kanalikulärer Cholestase sowie herdförmiger vakuoliger Zelldegeneration mit nur wenigen Einzelzellnekrosen (Abbildung 1). Dieser Befund war mit einer Leptospirose vereinbar. In der Silberfärbung konnte kein direkter Erregernachweis geführt werden. Für eine Malignität ergab sich kein Anhalt.

Die auswärts begonnene antibiotische Therapie war nach 4 Tagen abgesetzt worden. Aufgrund der deutlichen Entzündungsaktivität in der Leber begannen wir am 8. Tag sicherheitshalber erneut mit einer Antibiotikatherapie (Penicillin G, dreimal $2 \mathrm{Mega} / \mathrm{d}$ ) für 6 Tage.

Der Patient blieb fieberfrei; durch kontinuierliche Hämofiltration bzw. Hämodialyse wurden die Kreatininkonzentration auf $4,9 \mathrm{mg} / \mathrm{dl}$ und der Harnstoffwert auf $130 \mathrm{mg} / \mathrm{dl}$ gesenkt. Ebenso war die Gesamtbilirubinkonzentration deutlich rückläufig $(4,4 \mathrm{mg} / \mathrm{dl})$. Während des Aufenthaltes auf der Intensivstation trat mehrmals Nasenbluten auf, das jedoch durch Tamponaden gut beherrscht werden konnte.

Auf der Normalstation gingen die Nierenretentions- und die Bilirubinwerte unter symptomatischer Therapie mit anfänglich Dopamin in der Dosis von $3 \mu \mathrm{g} / \mathrm{kg} \cdot \min$ und Furosemid $(40 \mathrm{mg} / \mathrm{d})$ weiter zurück. Die Thrombozytenzahl stieg konti-

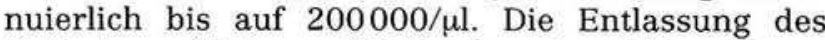
Patienten erfolgte am 36. Tag in gutem Allgemeinzustand. 


\section{Diskussion}

Leptospiren sind ubiquitäre Keime, die unter bestimmten klimatischen Bedingungen und optimalen Boden- und Gewässerverhältnissen (Temperaturen über $22^{\circ} \mathrm{C}$, neutrale bis alkalische $\mathrm{pH}$ Werte sowie feuchtes Milieu mit niedrigem Salzgehalt) mehrere Wochen überleben können. Zumeist beträgt ihre Überlebenszeit jedoch nur 6-48 Stunden am Boden oder im Gewässer. Erregerreservoire sind in erster Linie Nagetiere wie Ratten und Mäuse, aber auch Rinder, Schweine, Ziegen, Hamster und Hunde können asymptomatische Träger dieser Keime sein. Bei einigen Tieren wie dem Waschbären und dem Fuchs werden Infektionsraten bis $50 \%$ angegeben.

Die Übertragung erfolgt durch Kontakt mit erregerhaltigem Urin, Gewebe oder Blut von Tieren, welche die Leptospiren als Wirt in verschiedenen Organsystemen beherbergen, oder durch Kontakt mit infiziertem Wasser, Erde oder Pflanzen. Eintrittspforte sind Hautverletzungen oder die Schleimhäute der Augen, des Nasenrachenraumes oder der Vagina $(7,8,12,13)$. Auch bei unserem Patienten dürfte eine kleine Hautverletzung im Bereich der rechten Hand die Eintrittspforte gewesen sein. In der Woche vor der stationären Aufnahme hatte er mit Fallen Ratten gefangen und war dabei vermutlich mit infiziertem Rattenurin in Berührung gekommen.

Nach einer Inkubationszeit von $7-13$ Tagen zeichnet sich die Leptospirose zunächst durch eine Vielfalt unspezifischer Symptome aus. Dazu gehören Fieber, Schüttelfrost, Kopfschmerzen, Konjunktivitis, rezidivierende Epistaxis, Myalgien, Übelkeit, Erbrechen und Durchfälle. Selten treten auch Hämoptysen im Rahmen pulmonaler Blutungen und ein akutes Lungenversagen (ARDS) mit entsprechend schwerem Verlauf auf $(2,3,6,11,13,15)$.

Diese erste Phase, die in der Regel 4-9 Tage dauert, wird auch als leptospirämische Phase bezeichnet. Man kann während dieser Zeit die Mikroorganismen im Blut und im Liquor nachweisen, und es kann zu einer Besiedelung nahezu aller Gewebe kommen. Nach einer relativ symptomarmen Periode von 1-3 Tagen, in der das Fieber zurückgeht und sich der Allgemeinzustand bessert, folgt dann die zweite Krankheitsphase, auch Immunphase genannt. Hier treten erstmals IgM-Antikörper im Serum auf. Gleichzeitig verschwinden die Leptospiren aus dem Blut und den meisten Geweben. In dieser Phase kann es erneut zu einem Fieberanstieg mit meningealen Reizerscheinungen bis hin zum Vollbild einer Meningitis mit Liquorpleozytose kommen. Aber auch bei symptomlosen Patienten findet man in bis zu 90\% der Fälle eine Pleozytose $(3,7$, 13). Weitere klinische Erscheinungsbilder dieser Phase können nahezu die gesamte Palette der neurologischen Erkrankungen umfassen (Enzephalitiden,
Spastik, Paralyse, Neuritiden, Myelitiden). Diese Krankheitsperiode dauert zwischen 4 und 30 Tagen und endet in der Regel mit der Rekonvaleszenz des Patienten; bis zu 35\% der Erkrankten bleiben in der zweiten Phase symptomfrei $(7,8,12,13)$.

Unter dem Krankheitsbild des Morbus Weil (rund 10\% der Fälle) versteht man den schweren Verlauf einer Leptospireninfektion mit cholestatischem Ikterus, eingeschränkter Nierenfunktion, Bewußtseinstrübung, Kreislaufkollaps, Status febrilis, seltener mit Hämolyse und Thrombozytopenie. Generell ist hierbei ein Befall aller Organe möglich. Im Gegensatz zur früheren Definition des Morbus Weil, von dem nur dann gesprochen wurde, wenn eine Infektion mit Bakterien der Serogruppe Leptospira interrogans varietas icterohaemorrhagiae bestand, weiß man heute, daß eine schwere Leptospirose auch durch Erreger anderer Serogruppen verursacht werden kann.

Die erste Krankheitsphase ähnelt der beschriebenen anikterischen Form. Die typischen Erscheinungsbilder des Morbus Weil beginnen ab dem 3.-6. Krankheitstag; dabei herrschen die Manifestationen an Leber und Nieren vor. Erst in der zweiten Phase entwickelt sich das Vollbild dieser Erkrankung. Klinisch-chemisch fällt vor allem ein exzessiv erhöhter Bilirubinwert auf, der hauptsächlich auf einen Anstieg des direkten Bilirubins zurückzuführen ist.

Die Transaminasen und Cholestaseparameter sind weitgehend normal oder gering erhöht. Zurückzuführen ist dies auf eine Ausscheidungsstörung der Hepatozyten in zentrolobulären Leberarealen $(4,5)$. Die Nierenfunktionsstörung zeigt sich in einer Proteinurie, Pyurie und Hämaturie. Oftmals entwickelt sich eine Oligurie oder Anurie im Sinne eines akuten Nierenversagens (histologisch oft dem Bild der akuten Tubulusnekrose entsprechend). Weitere Erscheinungsbilder der schweren Leptospirose sind Hämorrhagien wie Epistaxis, Hämoptoe oder gastrointestinale Blutungen.

Wie aus dem dargestellten Krankheitsverlauf ersichtlich ist, lief die leptospirämische Phase bereits im auswärtigen Krankenhaus ab, und der Patient kam erst mit dem Vollbild der zweiten Phase eines Morbus Weil in unsere Klinik. Aufgrund der vielfältigen und unspezifischen Symptome am Beginn der Krankheit ist oftmals, wie bei unseren Patienten, eine frühzeitige Diagnose nicht möglich.

Die Prognose der Leptospirose ist bei anikterischem Verlauf gut, die Patienten erholen sich rasch. Bei den schweren Verläufen hängt der Ausgang der Erkrankung von der Virulenz der Erreger, vom Alter der Patienten, deren Allgemeinzustand und dem Auftreten von Ikterus, Nierenversagen sowie Beteiligung der Atemwege ab. Ebenso spielen 
Blutungskomplikationen eine wichtige Rolle. Hier wurde eine Letalität von fast $50 \%$ angegeben (13). Durch den frühzeitigen Einsatz der Dialyse konnte die Letalität in diesen Fällen erheblich gesenkt werden. Die Langzeitverläufe der Nierenschädigung und der Leberbeteiligung sind in der Regel gutartig $(9,12$, 13). Als Residuen werden in seltenen Fällen tubuläre Funktionsstörungen beobachtet. Die Leberveränderungen bilden sich über mehrere Wochen zurück ( 4 , $12,13)$.

Die Behandlung der Leptospirose beinhaltet Flüssigkeits- und Elektrolytkontrolle, Dialyse und parenterale Ernährung bei Nierenversagen und in selteneren Fällen bei massiver Hyperbilirubinämie Austauschtransfusionen. Eine antibiotische Therapie mit Penicillin G (1-2 Mega-E alle $6 \mathrm{~h}$ ) oder Doxycyclin $(200 \mathrm{mg}-2 \mathrm{~g} / \mathrm{d})$ über 1 Woche ist nur dann wirksam, wenn sie innerhalb von 4-7 Tagen nach Krankheitsbeginn einsetzt $(12,13)$.

Zusammenfassend ist $\mathrm{zu}$ sagen, $\mathrm{da} \beta$ bei unklarer Symptomatik mit Ikterus auch in unseren Regionen die Leptospireninfektion in die differentialdiagnostischen Erwägungen einbezogen werden muß, obwohl sie hier, wie aus den genannten Zahlen hervorgeht, eher eine seltene Infektionskrankheit ist.

\section{Literatur}

1 Alexander, A. D.: Leptospira. In Braude, A. I. (Ed.): Infectious Diseases and Medical Microbiology, 2. ed., International Textbook of Medicine (Samiy-Smith: Wyngaarden 1986), 381-385.

2 Allen, P., S. Raftery, D. Phelan: Massive pulmonary haemorrhage due to leptospirosis. Intensive Care Med. 15 (1989), 322-324.

3 Berman, St., et al.: Sporadic anicteric leptospirosis in south Vietnam. A study in 150 patients. Ann. intern. Med. 79 (1973), 167-173.

4 Bhamarapravati, N., V. Viranuvatti, U. Tuchinda, D. Boonnag, S. W. Nye: Liver changes in leptospirosis. A study of needle biopsies in twenty-two cases. Amer. J. Proctol. 17 (1966), 480-487.

5 Dooley, J. R., K. G. Ishak: Leptospirosis. In Binford, C. H., D. H. Connor (Ed.): Pathology of Tropical and Extraordinary Diseases (Armed Forces Institute of Pathology: Washington 1976), 101-106.

6 Edwards, C. N., G. D. Nicholson, T. A. Hassell, C. O. Everard, J. Callender: Leptospirosis in Barbados. A clinical study. West Indian med. J. 39(1) (1990), 27-34.

7 Edwards, G. A.: Leptospirosis. In Braude, A. I. (Ed.): Infectious Diseases and Medical Microbiology, 2. ed., International Textbook of Medicine (Samiy-Smith: Wyngaarden 1986), $1543-1547$.

8 Farrar, W. E.: Leptospira species (Leptospirosis). In Mandell, G. L., R. G. Douglas, J. E. Bennett (Ed.): Principles and Practice of Infectious Diseases, 3. ed. (Churchill Livingstone: New York-Edinburgh-London-Melbourne 1990), 1813-1816.

9 Kennedy, N. D., C. D. Pusey, D. J. Rainford, A. Higginson: Leptospirosis and acute renal failure - clinical experiences and a review of literature. Postgrad. med. J. 55 (1979), 176-179.

10 Macura, T., R. Jarczyk: Weil's disease complicated by subarachnoid hemorrhage and myocardial infarction. Wiad. lek. $43(13)(1990), 685-687$.

11 Park, S. K., S. H. Lee, Y. K. Rhee, S. K. Kang, K. J. Kim, M. C. Kim, K. W. Kim, W. H. Chang: Leptospirosis in Chonbuk Province of Korea in 1987. A study of 93 patients. Amer. J. med. Hyg. 41(3) (1989), 345-351.

12 Sanford, J. P.: Leptospirosen. In Straub, P. W.: Harison's Prinzipien der inneren Medizin (Schwabe: Basel 1989), 773-777.

13 Sitprija, V.: Leptospirose. In Warrell, D. A.: Infektionskrankheiten (Edition Medizin: Weinheim 1990), 404-410.

14 Statistisches Bundesamt: Fachserie 12: Gesundheitswesen, Reihe 2: Meldepflichtige Krankheiten (Metzler-Poeschel: Kusterdingen 1989 und 1990).

15 Tulli, G., R. Gabini, M. Feri, P. Maurizi, S. Nannoni, G. Vignali: Multiple organ failure syndrome in infection caused by icterohaemorrhagic leptospira. Description of a clinical case. Minerva anestesiol. 55(10) (1989), 423-426.

Dr. W. F. Notheis, Privatdozent Dr. B. K. Krämer,

Prof. Dr. E. P. Kromer, Prof. Dr. A. J. G. Riegger

Klinik und Poliklinik für Innere Medizin II der Universität

Prof. Dr. H.-G. Leser

Klinik und Poliklinik für Innere Medizin I der Universität

Prof. Dr. J. Rüschoff

Pathologisches Institut der Universität

Franz-Josef-Strauß-Allee 11

93053 Regensburg 